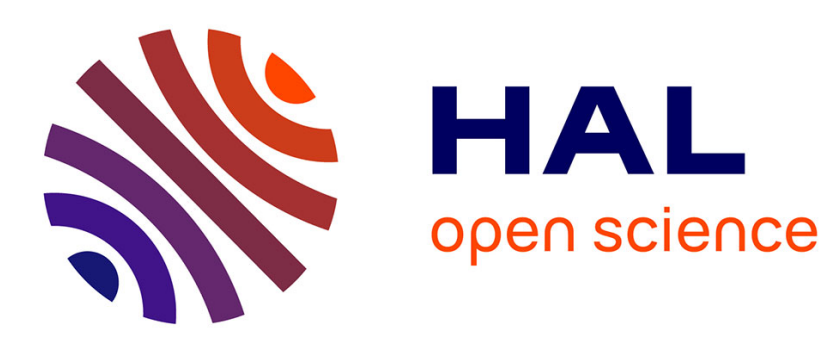

\title{
Les déterminants d'un apport protidique faible chez les personnes âgées dépendantes
}

Claire Sulmont-Rossé, Virginie van Wymelbeke

\section{To cite this version:}

Claire Sulmont-Rossé, Virginie van Wymelbeke. Les déterminants d'un apport protidique faible chez les personnes âgées dépendantes. Cahiers de Nutrition et de Diététique, 2019, 54 (3), pp.180-189. 10.1016/j.cnd.2019.02.003 . hal-02620667

\section{HAL Id: hal-02620667 https://hal.inrae.fr/hal-02620667}

Submitted on 4 Feb 2021

HAL is a multi-disciplinary open access archive for the deposit and dissemination of scientific research documents, whether they are published or not. The documents may come from teaching and research institutions in France or abroad, or from public or private research centers.
L'archive ouverte pluridisciplinaire HAL, est destinée au dépôt et à la diffusion de documents scientifiques de niveau recherche, publiés ou non, émanant des établissements d'enseignement et de recherche français ou étrangers, des laboratoires publics ou privés. 


\title{
LES DÉTERMINANTS D'UN APPORT PROTIDIQUE FAIBLE CHEZ LES PERSONNES ÂGÉES DÉPENDANTES «
}

\author{
Claire Sulmont-Rossé ${ }^{1}$ \& Virginie Van Wymelbeke $e^{1,2}$
}

${ }^{1}$ Centre des Sciences du Goût et de l'Alimentation, AgroSup Dijon, CNRS, INRA, Université Bourgogne Franche-Comté, F-21000 Dijon.

${ }^{2}$ Centre Hospitalier Universitaire de Dijon Bourgogne François Mitterrand, Centre Gériatrique Champmaillot, Unité de Recherche du Pôle Personnes Âgées, F-21000 Dijon.

خैarticle ayant fait l'objet d'une conférence lors des JFN2017

\section{CORRESPONDANCE}

Claire Sulmont-Rossé, 17 rue Sully, BP 86510, 21065 Dijon Cedex, +33 3806932 71, claire.sulmont-rosse@,inrae.fr

\section{RÉSUMÉ EN FRANCAIS (1110 SIGNES)}

Le vieillissement est associé à de nombreux changements physiologiques, sensoriels, psychologiques et sociologiques susceptibles d'avoir un impact délétère sur la prise alimentaire et le statut nutritionnel de la personne âgée. La situation est particulièrement préoccupante en institution où une large majorité de personnes âgées ne satisfait pas à ses besoins caloriques et protidiques, notamment du fait d'une consommation insuffisante des plats protidiques servis au déjeuner et au dîner. Des relevés alimentaires réalisées en EHPAD ont montré que $83 \%$ des résidents ne satisfaisaient pas à leurs besoins caloriques et protidiques ( $43 \%$ présentaient un apport inférieur aux $2 / 3$ des apports conseillés). Le maintien des apports protidiques et notamment de la consommation de viande en institution se heurte d'une part aux croyances des personnes âgées elles-mêmes ("Â mon âge, je n'ai plus besoin de manger autant de viande !») mais également à la maitrise souvent difficile de la qualité des plats protidiques servis en restauration collective (assaisonnement, cuisson, maintien en température...).

Mots clefs : Senior ; institution ; prise alimentaire ; appétit ; viande

\section{TITLE}

Determinants of a low protein intake in the institutionalized elderly people

\begin{abstract}
Ageing is associated with several physiological, sensory, psychological and sociological changes that may have a negative impact on food intake and nutritional status in the elderly population. This situation is particularly worrying in nursing homes where a large majority of elderly people does not satisfy caloric and protein requirements, notably because of insufficient
\end{abstract}


consumption of protein dishes at lunch or at dinner. 24-hr dietary records carried out in several nursing homes showed that $83 \%$ of elderly people did not cover their caloric and protein needs ( $43 \%$ had a caloric and/or protein intake lower than $2 / 3$ of the Daily Recommended Allowance). Sustaining protein intake and meat consumption in institutions have to face the elderly's beliefs ("At my age, I no longer need to eat so much meat!"), but also the difficult challenge of providing good quality protein dishes in catering services (seasoning, cooking, control of temperature...).

Keywords : Older adults; nursing home; food intake; appetite; meat 


\section{INTRODUCTION}

En France, comme dans la plupart des pays développés, la proportion de personnes âgées dans la population est en pleine croissance : 24 millions de personnes de plus de 60 ans en 2060 contre environ 17 millions en 2016. Le vieillissement de la population se caractérise par une forte progression des très âgés ( +85 ans : 1,3 million aujourd'hui, 5,4 millions en 2060 ; [1] et du nombre de personnes en perte d'autonomie (deux fois plus de personnes en 2060 ; [2]). Dans ce contexte de population vieillissante, l'un des enjeux majeurs de notre société actuelle est de permettre à tout un chacun de « bien vieillir ». Bien qu'avoir une «bonne alimentation » soit reconnu comme un facteur clef d'un vieillissement réussi, la dénutrition reste une menace importante pour la santé, l'autonomie et le bien-être des personnes âgées [3-6]. La dénutrition, pathologie reconnue du sujet âgée, correspond à un déficit des apports nutritionnels, en termes de calories et/ou de nutriments. Ses conséquences, multiples, comprennent entre autres la fonte musculaire et une altération des défenses de l'organisme. Sans prise en charge, elle augmente le risque de chutes, de fractures, d'épisodes pathologiques, d'hospitalisation [6-10]. Elle induit ou aggrave un état de fragilité et de dépendance, pour in fine affecter la qualité et l'espérance de vie des personnes âgées [11]. Selon la revue de la Haute Autorité de Santé [12], 4 à 10\% des personnes âgées vivant à domicile seraient dénutries, cette proportion atteignant 15 à $38 \%$ en Etablissement d'Hébergement pour Personnes Âgées Dépendantes (EHPAD) et 30 à $70 \%$ à l'hôpital.

Contrairement aux idées reçues, les besoins nutritionnels diminuent peu avec l'âge et sont parfois même supérieurs aux besoins de sujets plus jeunes. En ce qui concerne les apports énergétiques, l'EFSA (European Food Safety Authority) recommande un apport de 2000 à 2600 pour les personnes de 30 à 39 ans, de 2000 à 2500 pour les personnes de 50 à 59 ans et de 1800 à 23000 pour les personnes de 70 à 79 ans [13]. En ce qui concerne les apports en protéines, des travaux récents pilotés par le groupe PROT-AGE [14] et par l'ESPEN [15] montrent que les personnes âgées ont besoin d'un apport protéique plus important que des sujets plus jeunes pour rester en bonne santé, préserver leurs capacités et lutter contre les infections. En effet, le vieillissement s'accompagne de changements dans le métabolisme protéique, notamment de l'apparition d'une résistance à la stimulation de la synthèse protéique musculaire par la prise du repas [16]. En conséquence, l'apport quotidien en protéines doit être de 1 à 1,2 g de protéines par kilo de poids corporel par jour pour une personne de plus de 60 ans en bonne santé contre 0,8 à $1 \mathrm{~g}$ par kilo de poids corporel chez les sujets plus jeunes $[14,15]$.

\section{LA PRISE ALIMENTAIRE CHEZ LES SENIORS}

A quel âge devient-on senior? De fait, la notion même de « senior » est très variable. Pour les professionnels du marketing, un individu devient senior dès lors qu'il a plus de 50 ans. Pour les pouvoirs publics, sont considérés comme seniors les 60-65 ans (âges d'accès à certaines prestations sociales). Pour les professionnels de santé, il s'agit des plus de 70 ans, parce que c'est en moyenne à 73 ans que survient le premier accident de santé. Enfin, Maître [17] a montré que l'âge de 80 ans constituait une charnière au-delà duquel une fragilité nutritionnelle semble s'installer. Dans cet article (et sauf précision contraire), nous avons considéré comme « senior » toute personne de plus de 65 ans.

\section{L'appétit des seniors}

En 2006, l'Etude Nationale Nutrition Santé menée conjointement par l'Institut de Veille Sanitaire (IVS), l'Université Paris 13 et la Caisse Nationale d'Assurance Maladie (CNAM) a 
montré que chez les hommes, la prise énergétique passait de $2400 \mathrm{kcal} / \mathrm{jour}$ chez les $18-29$ ans à $2000 \mathrm{kcal} /$ jour chez les 55-74 ans [18]. Chez les femmes, pour les mêmes tranches d'âge, la prise énergétique diminuait de $1700 \mathrm{kcal} /$ jour à $1500 \mathrm{kcal} /$ jour. Toujours en France, Bon et al. [19] ont montré qu'à l'hôpital et en maison de retraite, la prise calorique moyenne atteignait à peine $1500 \mathrm{kcal}$ par jour - seuil en dessous duquel il est difficile de couvrir les besoins nutritionnels de l'organisme [20]. Cette baisse des quantités consommées s'accompagne d'une baisse de la variété alimentaire [21], en particulier pour les personnes âgées vivant en maison de retraite [22], ainsi qu'une réduction des apports en nutriments et en micronutriments [11, 23]. Ce déséquilibre entre besoins nutritionnels et apports alimentaires prédispose les personnes âgées à une perte de poids involontaire et augmente le risque nutritionnel.

Le processus de vieillissement, même lorsqu'il se déroule normalement, est associé à de nombreuses modifications d'ordre physiologique, sensorielle, psychologique et sociologique susceptibles d'avoir un impact sur la prise alimentaire et le statut nutritionnel de la personne âgée [24].

\section{Facteurs liés au vieillissement}

D'un point de vue physiologique, l'âge s'accompagne de changements gastro-intestinaux tels qu'un ralentissement de la vidange gastrique, du transit intestinal, une élévation du niveau de base d'hormones anorexigènes [25] et une diminution du métabolisme de base [26]. Ces modifications contribuent à la dérégulation de l'appétit et à l'altération des mécanismes de compensation se mettant normalement en place après une période de sous ou de suralimentation [27, 28].

Par ailleurs, le vieillissement s'accompagne fréquemment de changements importants dans la sphère orale, tels que la perte de dents [29], la diminution du flux salivaire [30] ou l'apparition de troubles de la déglutition [31]. Ces altérations du statut bucco-dentaire peuvent conduire les personnes âgées à éviter la consommation d'aliments difficiles à mâcher tels que les fruits et les légumes crus ainsi que la viande [32-34]. De plus, la baisse de l'efficacité masticatoire et du flux salivaire altère la dégradation des aliments et la formation du bol alimentaire, mécanismes qui contribuent normalement à la libération des nutriments et micronutriments dans le tractus digestif [35].

Enfin, de nombreuses études ont montré que l'âge s'accompagne d'une altération des sens gustatif et olfactif, ce qui se traduit par un déclin de la capacité à percevoir les odeurs ou les saveurs des aliments [36-40]. Plusieurs auteurs ont montré que le déclin des capacités chimiosensorielles pouvait s'accompagner d'un déclin de l'appétit [41], de l'intérêt pour l'alimentation [42] ou de la variété alimentaire [43]. Toutefois, l'impact du déclin des capacités chimiosensorielles sur la prise alimentaire reste controversé à ce jour [41, 44]. En parallèle, une altération du mécanisme de rassasiement sensoriel-spécifique a été observée chez les personnes âgées [45], en particulier chez les personnes âgées institutionnalisées [46]. Le rassasiement sensoriel spécifique correspond à une diminution du plaisir induit par un aliment au cours de la consommation de cet aliment, ce qui nous incite à varier notre alimentation.

\section{Facteurs liés à la trajectoire de vie}

Au-delà des modifications propres au vieillissement, la vie d'une personne âgée est marquée par des "moments de rupture " (veuvage, survenue d'une pathologie, dépendance...) susceptibles de bouleverser ses habitudes de vie et en particulier ses habitudes alimentaires [47]. Cardon et Gojard [48] ont par exemple montré que la délégation à un tiers (membre de la famille 
ou aide professionnelle) d'une partie des activités alimentaires suite à l'apparition d'incapacités physiques ou psychiques entraînait une diminution de la variété alimentaire. Cardon [47] a également montré que le veuvage modifiait les habitudes alimentaires de la personne avec une disparition des plats « familiaux » porteurs de convivialité et de sociabilité (par exemple, les pâtisseries maison) et chez les veufs, une augmentation de la consommation de plats préparés ou surgelés.

En parallèle de ces changements socioéconomiques, la survenue de pathologies affecte également la prise alimentaire des personnes âgées [49]. Un épisode pathologique aigü (infection, fracture, cancer), une intervention chirurgicale, une hospitalisation, la fièvre, la douleur et la polymédication sont autant de facteurs associés au risque nutritionnel chez les personnes âgées $[12,24]$. De nombreux médicaments sont connus pour entraîner une baisse d'appétit [50], une modification du transit intestinal [50], une modification des fonctions buccodentaires [51-54] ou une altération des capacités chimiosensorielles [55-57]. Enfin, les maladies neurodégénératives telles que la maladie d'Alzheimer sont souvent associés à des troubles associées à la prise alimentaire : difficulté de se souvenir de l'heure du repas et des repas précédents, troubles de la préhension et de la déglutition, difficulté à rester à table [58$60]$.

En résumé de cette partie, même lorsqu'il se déroule normalement, le vieillissement peut s'accompagner d'une baisse de la prise alimentaire. Cette baisse de la prise alimentaire est induite par un vieillissement des fonctions physiologiques liées à l'alimentation (perception sensorielle, mastication, salivation, digestion) mais peut être modulée en fonction de l'histoire de vie de chaque individu (veuvage, modification des revenus, survenue d'une pathologie...). En particulier, cette perte d'appétit est majorée lorsque la personne âgée est amenée à déléguer tout ou partie de son alimentation à un tiers du fait de l'apparition d'incapacités physiques ou cognitives.

\section{LE CAS PARTICULIER DU MANGEUR ÂGÉ EN INSTITUTION}

L'entrée en EHPAD fait généralement suite à une perte d'autonomie de la personne âgée : apparition d'une incapacité physique ou psychique, veuvage, etc. Qu'il soit volontaire ou non, l'emménagement en EHPAD marque une rupture dans la vie de la personne âgée en modifiant ses habitudes, son environnement physique et social. En plus de s'adapter à la vie en collectivité, la personne doit également s'adapter à des règles édictées par de tierces personnes, notamment en ce qui concerne son alimentation. Dès lors, la personne âgée est confrontée aux pratiques culinaires, aux habitudes alimentaires, aux connaissances (ou croyances) nutritionnelles, voire aux préférences alimentaires des personnes en charge de la nourrir [33]. Il est donc possible que ces changements cristallisent progressivement de nouvelles habitudes alimentaires, parfois en rupture avec les capacités et préférences de la personne âgée, et soient à l'origine de déséquilibres alimentaires [49, 61, 62]. Des travaux menés par le sociologue Philippe Cardon et l'anthropologue Emmanuel Soutremon ont montré que la cuisine était perçue comme une " boîte noire » par les résidents des maisons de retraite [63]. Déléguer tout ou partie de son alimentation à un tiers crée une distanciation entre la personne âgée et son alimentation, susceptible de générer un désengagement et contribuer à la perte d'appétit [6466]. L'étude de Pouyet et al [33] souligne d'ailleurs l'importance d'avoir des plats perçus comme familiers par les résidents, dans un contexte où ces derniers n'ont pas ou très peu d'emprise sur le choix des menus et des recettes utilisées. "Dans un environnement tel que l'EHPAD, qui constitue une rupture des habitudes de vie, l'alimentation reste un moyen d'affirmer son identité » concluent les auteurs. 


\section{Le petit mangeur âgé en institution}

Van Wymelbeke et Sulmont-Rossé [67] ont recruté 88 personnes âgées vivant en institution (66 à 100 ans ; 86,6 \pm 0.8 d'âge moyen; 70\% de femmes) et pesé pendant 24 heures tous les aliments et toutes les boissons consommées par ces personnes. La composition nutritionnelle a été déterminée à l'aide de la table CIQUAL (Version 2016 ; https://ciqual.anses.fr/). Pour chaque sujet, nous avons calculé le rapport entre la prise calorique ou protidique du sujet et les Apports Journaliers Conseillés (AJC calories : $30 \mathrm{kcal} / \mathrm{kg}$ de poids corporel / jour pour ; AJC protéines : $1,2 \mathrm{~g} / \mathrm{kg}$ de poids corporel / jour). Les résultats ont montré que $13 \%$ seulement des personnes âgées vivant en institution satisfaisaient à leurs besoins caloriques et protidiques (apport calorique et protidique $\geq 100 \%$ du besoin). Ces mangeurs ont été qualifiés de «mangeurs normaux ». Parmi les autres participants, nous avons distingué les « petits mangeurs » $(41 \%$ de l'effectif), qui présentaient un apport calorique et/ou protidique inférieur à $100 \%$ mais supérieur au 2/3 des apports recommandés, et les «tout petits mangeurs » $(46 \%)$, qui présentaient un apport calorique et/ou protidique inférieur aux $2 / 3$ des apports recommandés (Tableau 1). Dans un second temps, nous avons trié les consommations en 10 catégories en fonction de leur composition nutritionnelle, de la taille de portion usuelle et du moment de consommation : produit céréalier du petit-déjeuner (e.g. pain), viande ou poisson du déjeuner, plat principal du dîner (e.g., lasagne, quiche), fromage, produit laitier frais (e.g. yaourt, fromage blanc), soupe, légume et fruit cuits, légume et fruit crus, produit sucré consommé au repas (e.g., tarte, gâteau), produit sucré consommé en collation (e.g., madeleine, biscuit). Le tableau 2 présente les moyennes des quantités ingérées pour chaque catégorie d'aliment et profil de mangeur. Les résultats montrent que les «tout petits mangeurs » se caractérisent par une consommation moindre de produits céréaliers au petit-déjeuner, de viande ou de poisson au déjeuner et de plat principal au dîner.

\section{La consommation de VPO (Viande, Poisson, (Euf) en EHPAD}

Sulmont-Rossé et all [68] ont évalué la fréquence de consommation hebdomadaire de 21 catégories d'aliments dont la viande, le poisson, le jambon blanc et les œufs chez des personnes âgées de plus de 65 ans, en distinguant les personnes âgées autonomes $(n=182$; âge : $M=72,6$ $(S E=0,4) ; 52 \%$ de femmes), les personnes bénéficiant d'une aide à domicile pour les courses ou la préparation des repas ( $n=91$; âge : $M=83,5(S E=0,8) ; 78 \%$ de femmes), les personnes bénéficiant d'un service de portage de repas à domicile ( $n=108$; âge : $M=83,9(S E=0,7) ; 65 \%$ de femmes) et les personnes vivant en $\operatorname{EHPAD}(n=120$; âge : $M=85,8(S E=0,6) ; 70 \%$ de femmes). Les résultats montrent une légère augmentation de la fréquence de consommation de viande chez les personnes âgées vivant en EHPAD (environ 6 fois par semaine) par rapport aux personnes dépendantes à domicile (environ 5 fois par semaine) (Tableau 3). De même, on peut observer une légère augmentation de la fréquence de consommation de poisson chez les personnes âgées dépendantes d'un service de restauration collective (portage de repas, EHPAD), probablement imputable aux règles nutritionnelles qui régissent les plans alimentaires de ces structures. Enfin, il est intéressant de noter la plus forte consommation de jambon chez les personnes âgées aidées à domicile : 20\% de ces personnes déclarent consommer du jambon au moins une fois par jour contre seulement $7 \%$ des personnes bénéficiant d'un portage et $4 \%$ des personnes vivant en EHPAD

En parallèle de ces travaux, nous avons mesuré les quantités de viande ou de poisson ingérées au déjeuner dans une situation de consommation ad libitum dans le cadre de plusieurs études menées en EHPAD[69-71] . Dans toutes ces études, les participants recevaient une portion de viande ou de poisson correspondant aux grammages préconisés par le GEMRCN [72] pour la restauration collective des personnes âgées, soit 100g pour la viande et le poisson. Un second 
service était systématiquement proposé aux participants ayant fini leur assiette (la portion servie était alors de $50 \mathrm{~g}$ de viande ou de poisson), et parfois même un troisième service. Les résultats montrent que quelle que soit l'étude, la moyenne des quantités de viande ou de poisson consommées au déjeuner par les résidents d'EHPAD est largement inférieure aux recommandations (Tableau 4). Cependant, ces résultats montrent également une très forte variabilité interindividuelle. La figure 1 présente la consommation de blanquette de veau relevée pour chaque participant dans le cadre de l'étude de Van Wymelbeke et al. [71]. Seuls $12 \%$ des participants ont consommé $100 \mathrm{~g}$ de viande ou plus. A l'opposé, $36 \%$ des participants ont consommé moins de $50 \mathrm{~g}$ de viande.

En résumé de cette partie, l'entrée en institution peut modifier en profondeur le rapport d'une personne âgée à son alimentation. Cette dernière est désormais confrontée aux pratiques culinaires et aux préférences alimentaires des personnes en charge de la nourrir, ainsi qu'aux normes en matière d'hygiène et sécurité alimentaire et d'équilibre nutritionnel qui prévalent dans ces établissements. Dans un contexte tel que l'EHPAD, Van Wymelbeke et SulmontRossé [67] ont montré que $84 \%$ des personnes âgées institutionnalisées ne satisfaisaient pas à leurs besoins protéiques, notamment du fait d'une consommation insuffisante de plat protéinés lors des repas principaux.

\section{CROYANCES ET PRÉFERENCES ASSOCIÉES A LA VIANDE CHEZ LE MANGEUR ÂGÉ}

Il n'est pas rare d'entendre des personnes âgées dire " Â mon âge, je n'ai plus besoin de manger autant que quand j'étais jeune " [73]. De fait, l'âge peut s'accompagner de l'apparition de croyances susceptibles d'affecter la prise alimentaire, et ce malgré les campagnes menées par les instances de santé publique. Une enquête qualitative pilotée par le Centre d'Information de la Viande (CIV) et l'ADIV a montré que $20 \%$ des femmes de plus de 65 percevait la viande comme un "aliment du travailleur », et de ce fait sous-estimaient leurs propres besoins en viande [74]. Plus récemment, nous avons interrogé 81 personnes âgées de plus de 65 ans vivant à domicile. Â la question «Après 70 ans, il faut manger moins de viande », 54\% ont répondu «faux ». $36 \%$ ont répondu «vrai» et $10 \%$ ne se prononçaient pas, témoignant d'une méconnaissance des besoins nutritionnels chez un nombre non négligeable de personnes âgées. Ainsi, au cours des entretiens menés auprès de ces personnes, les personnes âgées ont déclaré qu' "Un morceau de viande, ça suffit! " ou bien que "Pour les jeunes, il faut un peu plus de protéines. Nous, on n'est plus tout jeune... [...]. On ne grandit plus à notre âge!».

A ce jour, il est difficile d'évaluer dans quelle mesure les préférences évoluent avec l'âge (à notre connaissance, il n'existe aucune étude longitudinale sur ce thème). Dans l'enquête AUPALESENS, nous avons interrogé 559 personnes âgées de plus de 65 ans sur l'évolution de leur consommation de viande (rouge ou blanche) et de poisson [17]. Parmi les personnes interrogées, 463 étaient autonomes pour leur alimentation et 196 étaient dépendantes pour leur alimentation (aide pour les courses ou la préparation des repas, portage de repas à domicile, EHPAD). Qu'elles soient autonomes ou dépendantes, 73\% des personnes âgées interrogées déclaraient consommer moins de viande rouge que lorsqu'elles avaient 30-40 ans tandis que $50 \%$ déclaraient consommer plus de poisson (Figure 2). Parmi les personnes âgées autonomes déclarant avoir diminué leur consommation de viande rouge, $42 \%$ déclaraient « moins aimer la viande », 26\% déclaraient « limiter la viande pour des raisons de santé » et $17 \%$ déclaraient « avoir moins d'appétit ». En revanche, les personnes âgées dépendantes pour leur alimentation associaient essentiellement un changement de consommation au fait que certains aliments sont peu proposés (viande rouge : $63 \%$ des réponses ; viande blanche : 59\%) ou davantage proposés 
(volaille : 86\% ; poisson : 70\%) par les structures d'aide (Tableau 5). Il est intéressant de rapprocher ce résultat d'un autre résultat obtenu dans l'enquête AUPALESENS : 68\% des personnes âgées vivant en EHPAD ont dit être d'accord avec la proposition « Un rôti de bœuf saignant et bien tendre : j'adore ! » contre seulement $54 \%$ des personnes âgées vivant à domicile [17]. Il est probable que ce résultat reflète une envie forte pour un plat rarement inscrit au menu des EHPAD.

Des enquêtes de satisfaction sont régulièrement menées auprès des personnes âgées dépendantes d'un service de restauration collective pour leur alimentation. Ainsi, l'association nationale de défense des consommateurs et usagers (Consommation Logement Cadre de Vie) a mené une enquête auprès de 251 personnes bénéficiant d'un service de portage et de 1217 personnes en institution. Les résultats ont montré que plus de $80 \%$ des personnes donnaient une appréciation globale des repas satisfaisante [75]. Cependant, ces résultats sont à prendre avec recul comme le souligne le sociologue Jean-Pierre Corbeau. En effet, la génération enquêtée est une population peu exigeante (elle a connu les années de privation pendant la guerre) et il s'agit essentiellement de femmes, souvent peu habituées à exprimer un désaccord. De plus, les personnes âgées hésitent souvent à émettre des doléances à l'égard de services dont elles sont dépendantes. Enfin, si la satisfaction globale est satisfaisante, les réponses n'étaient pas exemptes de critiques. Les doléances portaient notamment sur le manque de variété des menus, le réchauffage des plats, l'assaisonnement insuffisant, le peu de produits préparés sur place à base de produits frais et la viande trop souvent cuisinée en sauce [75]. De fait, le plat principal qui comprend une composante protidique (viande, poisson...) et un accompagnement (légumes, féculents...) cristallise fréquemment les insatisfactions des personnes âgées dépendantes pour leur alimentation. Les personnes âgées expriment des attentes pour un plat « familial » pas toujours compatible avec les contraintes de la restauration collective ( $U$ Un bon pot-au-feu comme à la maison! »). C'est également un plat qui nécessite de bonnes compétences culinaires et une maîtrise soigneuse de la température pour être servi « chaud» et non " tiède ». Comme le rappelle Grégoire Maille, cuisinier, il est essentiel de développer des recettes, des techniques et des formations à destination du personnel de cuisine œuvrant en restauration collectives. On ne cuisine pas de la même façon dans un restaurant gastronomique et dans une cuisine centrale. Ceci nécessite également une bonne coordination entre personnel de cuisine et personnel de service : "Lorsqu'on parle de cuisine centrale, ce sont des centaines voire des milliers de repas confectionnés chaque jour. Pour autant, la viande doit être bien cuite, bien assaisonnée, etc. Mais ensuite, la remise en température peut assécher le produit. Les cuisiniers peuvent faire plein d'efforts en cuisine, mais si au moment du service, les produits sont montés dans un four à $200^{\circ}$ pendant 2 heures, le steak sera tout sec dans l'assiette du convive. » [76].

En conclusion de cette partie, la consommation de plats à base de protéines animales et notamment de viande en EHPAD se heurte aux attentes sensorielles (tendreté, jutosité, assaisonnement...) et culinaires (mode cuisson, avec ou sans sauce, température de service...) fortes des résidents, pas toujours compatibles avec les contraintes de la restauration collective [33].

\section{CONCLUSION}

En conclusion, le processus de vieillissement, même lorsqu'il se déroule normalement, est associé à de nombreux changements d'ordre physiologique, sensoriel, psychologique et sociologique susceptibles d'avoir un impact délétère sur la prise alimentaire et le statut nutritionnel de la personne âgée. La situation est particulièrement préoccupante en institution où une large majorité des personnes âgées ne satisfait pas à ses besoins caloriques et protidiques, 
notamment du fait d'une consommation insuffisante des composantes protidiques servies au déjeuner et au dîner : 3 personnes sur 4 ne couvrent pas leurs besoins protidiques. De fait, la prévalence de la dénutrition est particulièrement élevée en institution (15 à $38 \%$ selon la Haute Autorité de Santé, 2007 ; 20-68\% selon des travaux plus récents : [77-79]). Dans ce contexte, il est essentiel $i$. de mieux informer les personnes âgées et former le personnel des institutions aux besoins nutritionnels de la personne âgée, $i i$. d'augmenter l'appétence des plats protidiques servis en institution et iii. d'enrichir l'alimentation des petits mangeurs âgés, c'est-à-dire d'augmenter la densité nutritionnelle des aliments sans augmenter la taille de portions.

\section{REMERCIEMENTS}

Les programmes AUPALESENS (ANR-09-ALIA-011-02), RENESSENS (ANR-13-ALID0006-02) et ALIMASSENS (ANR-14-CE20-0003-01]) ont été financés par l'Agence Nationale de la Recherche. Ces travaux de recherche sont également soutenus par le Conseil Régional de Bourgogne, le Fond Européen pour le Développement Economique Régional (FEDER), ainsi que par les pôles de compétitivité VITAGORA et VALORIAL. Plus d'information sur : https://www2.dijon.inra.fr/senior-et-sens/index.php. Les auteurs remercient Eléonore Bole, Lydie Champion, Cyrielle de Fiorido, Valérie Feyen, Naël Haidar, Elina Houde, Hélène Joyer, Mailis Saint-Jalmes, Anna Sauget pour leur aide lors des relevés alimentaires ainsi qu'Eléonore Boyer pour l'enquête ALIMASSENS. Enfin, les auteurs remercient Isabelle Maître et Gilles Feron, amis et collaborateurs précieux des études cités dans cet article.

\section{CONFLIT D'INTERET}

Les auteurs déclarent n'avoir aucun conflit d'intérêt dans le cadre des travaux de recherche présentés dans cet article.

\section{BIBLIOGRAPHIE}

[1] Bellin L, Morin A-C, Perrel C, Pfister C. Projections de population à l'horizon 2060. INSEE 2010; 1320:

[2] Martial F, Naudy-Fesquet I, Roosz P, Tronyo J.Tableaux de l'économie française. Paris, France: Collection INSEE Références; 2014, p.

[3] Di Francesco V, Fantin F, Omizzolo F, Residori L, Bissoli L, Bosello O, et al. The anorexia of aging. Digestive Diseases 2007; 25:129-137.

[4] Leslie WS. Improving the dietary intake of frail older people. Proceedings of the Nutrition Society 2011; 70:263-267.

[5] Merrell J, Philpin S, Warring J, Hobby D, Gregory V. Addressing the nutritional needs of older people in residential care homes. Health \& Social Care in the Community 2012; 20:208-215.

[6] Rasheed S, Woods RT. Malnutrition and quality of life in older people: A systematic review and meta-analysis. Ageing Research Reviews 2013; 12:561566.

[7] Cederholm T, Nouvenne A, Ticinesi A, Maggio M, Lauretani F, Ceda GP, et al. The Role of Malnutrition in Older Persons with Mobility Limitations. Current Pharmaceutical Design 2014; 20:3173-3177.

[8] Corti MC, Guralnik JM, Salive ME, Sorkin JD. Serum-albumin level and physical-disability as predictors of mortality in older persons. Journal of the American Medical Association 1994; 272:1036-1042. 
[9] Raynaud-Simon A, Lesourd B. Malnutrition in the elderly. Clinical consequences. . La Presse Médicale 2000; 29:2183-2190.

[10] Wallace JI, Schwartz RS, Lacroix AZ, Uhlmann RF, Pearlman RA. Involuntary weight-loss in older outpatients - incidence and clinical-significance. Journal of the American Geriatrics Society 1995; 43:329-337.

[11] Ferry M.Conséquences globales de la dénutrition. In: Ferry M, Alix E, Brocker P, Constans T, Lesourd B, Pfitzenmeyer P, Vellas B, editors. Nutrition de la Personne Âgée (4 édition), Paris, France: Elsevier; 2012, p.

[12] Haute Autorité de Santé HAS.Stratégies de prise en charge en cas de dénutrition protéino-énergétique chez les personnes âgées. Recommendations d'Avril 2007; 2007, p.

[13] European Food Safety Authority E. Scientific opinion on dietary reference values for energy. EFSA Journal 2013; 11:3005.

[14] Bauer J, Biolo G, Cederholm T, Cesari M, Cruz-Jentoft AJ, Morley JE, et al. Evidence-based recommendations for optimal dietary protein intake in older people: a position paper from the PROT-AGE Study Group. J Am Med Dir Assoc 2013; 14:542-559.

[15] Deutz NE, Bauer JM, Barazzoni R, Biolo G, Boirie Y, Bosy-Westphal A, et al. Protein intake and exercise for optimal muscle function with aging: recommendations from the ESPEN Expert Group. Clin Nutr 2014; 33:929-936.

[16] Walrand S, Guillet C, Salles J, Cano N, Boirie Y. Physiopathological mechanism of sarcopenia. Clin Geriatr Med 2011; 27:365-385.

[17] Maître I. Perceptions sensorielles et préférences alimentaires des seniors : contribution au maintien du statut nutritionnel et à l'appréciation des produits. 2014;

[18] Institut de Veille Sanitaire. Etude nationale nutrition santé ENNS : Situation nutritionnelle en France en 2006 selon les indicateurs d'objectif et les repères du Programme National Nutrition Santé (PNNS). 2006; http://www.ladocumentationfrancaise.fr/var/storage/rapportspublics/074000748.pdf.

[19] Bon F, Manckoundia P, Pfitzenmeyer P, Van Wymelbeke V. Nutritional intervention during hospitalisation in malnourished patients. Journal of Aging Research and Clinical Practice 2012; 3:213-218.

[20] Dupin H, Hercberg S.Etablissement des apports nutritionnels conseillés. In: Hercberg S, Dupin H, Papoz L, Galan P, editors. Nutrition et Santé Publique, Paris, France: Tech Doc; 1985, p.

[21] Fanelli MT, Stevenhagen KJ. Characterizing consumption patterns by food frequency methods - core foods and variety of foods in diets of older americants. Journal of the American Dietetic Association 1985; 85:1570-1576.

[22] Brown EL. Factors influencing food choices in the elderly. Geriatrics 1976; 31:89-92.

[23] Drewnowski A, Shultz JM. Impact of aging on eating behaviors, food choices, nutrition, and health status. The Journal of Nutrition, Health \& Aging 2001; 5:7579.

[24] Morley JE. Decreased food intake with aging. Journals of Gerontology Series a-Biological Sciences and Medical Sciences 2001; 56:81-88.

[25] Hays NP, Roberts SB. The anorexia of aging in humans. Physiology \& Behavior 2006; 88:257-266.

[26] Clarkston WK, Pantano MM, Morley JE, Horowitz M, Littlefield JM, Burton FR. Evidence for the anorexia of aging: Gastrointestinal transit and hunger in 
healthy elderly vs young adults. American Journal of Physiology-Regulatory Integrative and Comparative Physiology 1997; 272:R243-R248.

[27] Roberts SB, Fuss P, Heyman MB, Evans WJ, Tsay R, Rasmussen $H$, et al. Control of food-intake in older men. Jama-Journal of the American Medical Association 1994; 272:1601-1606.

[28] Rolls BJ, Dimeo KA, Shide DJ. Age-related impairments in the regulation of food intake. Am J Clin Nutr 1995; 62:923-931.

[29] Auvray L, Doussin A, Le Fur P. Santé, soins et protection sociale en 2002. 2003; http://www.irdes.fr/Publications/Rapports2003/rap1509.pdf.

[30] Vandenberghe-Descamps M, Labouré H, Prot A, Septier C, Tournier C, Feron $\mathrm{G}$, et al. Salivary flow decreases in healthy elderly people independently of dental status and drug intake. Journal of Texture Studies 2016; 47:353-360.

[31] Puisieux F, d'Andrea C, Baconnier P, Bui-Dinh D, Castaings-Pelet S, Crestani $B$, et al. Troubles de la déglutition du sujet âgée et pneumopathies en 14 questions / réponses. Revue des Maladies Respiratoires 2009; 26:587-605.

[32] Kiesswetter E, Poggiogalle E, Migliaccio S, Donini LM, Sulmont-Rossé C, Feart $\mathrm{C}$, et al. Functional determinants of dietary intake in community-dwelling older adults: a DEDIPAC (DEterminants of Dlet and Physical ACtivity) systematic literature review. Public Health Nutrition 2018; 21:1886-1903.

[33] Pouyet V, Giboreau A, Cuvelier G, Benattar L. Les préférences culinaires des personnes âgées vivant en institution : facteurs d'appréciation sensoriels et cognitifs. Cahiers de Nutrition et de Diététique 2015; 50:271-279.

[34] Tada A, Miura H. Systematic review of the association of mastication with food and nutrient intake in the independent elderly. Archives of Gerontology and Geriatrics 2014; 59:

[35] Peyron MA, Woda A, Bourdiol P, Hennequin M. Age-related changes in mastication. Journal of Oral Rehabilitation 2017; 44:299-312.

[36] Doty RL, Shaman P, Appelbaum SL, Giberson R, Siksorski L, Rosenberg L. Smell identification: Changes with age. Science 1984; 226:1441-1443.

[37] Methven L, Allen VJ, Withers CA, Gosney M.Ageing and Taste. Cambridge, UK: Cambridge University Press; 2012, p.

[38] Mojet J, Christ-Hazelhof E, Heidema J. Taste perception with age: generic or specific losses in threshold sensitivity to the five basic tastes? Disponible online $2001 ; 26: 845-860$.

[39] Murphy C.Taste and smell in the elderly. In: Meiselman ML, Rivlin RS, editors. Clinical measurement of taste and smell, New York: Macmillan; 1986, p.

[40] Sulmont-Rosse C, Maitre I, Amand M, Symoneaux R, Van Wymelbeke V, Caumon $\mathrm{E}$, et al. Evidence for different patterns of chemosensory alterations in the elderly population: impact of age versus dependency. Disponible on-line 2015; 40:153-164.

[41] de Jong N, Mulder I, de Graaf C, van Staveren WA. Impaired sensory functioning in elders: The relation with its potential determinants and nutritional intake. Journal Gerontology Serie A Biological Sciences Medical 1999; 54:B324-B331.

[42] Duffy VB, Backstrand JR, Ferris AM. Olfactory dysfunction and related nutritional risk in free-living elderly women. Journal of the American Dietetic Association 1995; 95:879-884.

[43] Kremer S, Holthuysen N, Boesveldt S. The influence of olfactory impairment in vital, independently living older persons on their eating behaviour and food liking. Disponible on-line 2014; 38:30-39. 
[44] Griep MI, Verleye G, Franck AH, Collys K, Mets TF, Massart DL. Variation in nutrient intake with dental status, age and odour perception. European Journal of Clinical Nutrition 1996; 50:816-825.

[45] Rolls BJ, McDermott TM. Effects of age on sensory-specific satiety. American Journal for Clinical Nutrition 1991; 54:988-996.

[46] Drewnowski A, Henderson SA, Driscoll A, Rolls BJ. The dietary variety score: Assessing diet quality in healthy young and older adults. Journal of the American Dietetic Association 1997; 97:266-271.

[47] Cardon P, Gojard S. Les personnes âgées face à la dépendance culinaire : entre délégation et remplacement. Retraite et société 2009; 56:169-193.

[48] Cardon P, Gojard S. Les personnes âgées face à la dépendance culinaire : entre délégation et remplacement. Retraite et société 2009; 56:56-79.

[49] Huffman GB. Evaluating and treating unintentional weight loss in the elderly. Am Fam Physician 2002; 65:640-650.

[50] Coleman Y.Drug-Nutrient Interactions - the Manual. Hawthorn, Australia: Nutrition Consultants Australia; 2003, p.

[51] Bardow A, Nyvad B, Nauntofte B. Relationships between medication intake, complaints of dry mouth, salivary flow rate and composition, and the rate of tooth demineralization in situ. Archives of Oral Biology 2001; 46:413-423.

[52] Baric JM, Handelman SL. SALIVARY FLOW, MOUTH-DRYNESS AND HYPOSALIVATORY DRUG-USE IN INSTITUTIONALIZED ELDERLY. Journal of Dental Research 1986; 65:232-232.

[53] Johanson CN, Osterberg T, Lernfelt B, Ekstrom J, Birkhed D. Salivary secretion and drug treatment in four 70-year-old Swedish cohorts during a period of 30 years. Gerodontology 2015; 32:202-210.

[54] Thomson WM. Dry mouth and older people. Australian Dental Journal 2015; 60:54-63.

[55] Doty RL, Bromley SM. Effects of drugs on olfaction and taste. Otolaryngologic Clinics of North America 2004; 37:1229-1254.

[56] Henkin RI, Levy LM, Fordyce A. Taste and smell function in chronic disease: A review of clinical and biochemical evaluations of taste and smell dysfunction in over 5000 patients at The Taste and Smell Clinic in Washington, DC. American Journal of Otolaryngology 2013; 34:477-489.

[57] Schiffman SS.Drugs influencing taste and smell perception. In: Getchell TV, Doty RL, Bartoshuk LM, Snow JB, editors. Smell and taste in health and disease., New York: Raven Press; 1991, p.

[58] Beattie ERA, Algase DL, Song J. Keeping wandering nursing home residents at the table: improving food intake using a behavioral communication intervention. Aging \& Mental Health 2004; 8:109-116.

[59] Durnbaugh T, Haley B, Roberts S. Assessing problem feeding behaviors in midstage Alzheimer's disease. Geriatric Nursing 1996; 17:63-67.

[60] Norberg A, Athlin E. Eating problems in severely demented patients - issues and ethical dilemmas. Nursing Clinics of North America 1989; 24:781-789.

[61] Caradec V.Sociologie de la Vieillesse et du Vieillissement. Paris: Arnaud Colin; 2008, p.

[62] Ferry M, Sidobre B, Lambertin A, Barberger-Gateau P. The SOLINUT study: analysis of the interaction between nutrition and loneliness in persons aged over 70 years. J Nutr Health Aging 2005; 9:261-268.

[63] Cardon P. Le regard du sociologue sur la dépendance chez les seniors. Dijon, France: Colloque AUPALESENS 2013; November 26. 
[64] Christiansen $\mathrm{CH}$. Defining lives: Occupation as identity: An essay on competence, coherence, and the creation of meaning - The 1999 Eleanor Clarke Slagle lecture. American Journal of Occupational Therapy 1999; 53:547558.

[65] Gustafsson K, Andersson I, Andersson J, Fjellstrom C, Sidenvall B. Older women's perceptions of independence versus dependence in food-related work. Public Health Nursing 2003; 20:237-247.

[66] Phinney A, Chaudhury H, O'Connor DL. Doing as much as I can do: The meaning of activity for people with dementia. Aging \& Mental Health 2007; 11:384-393.

[67] Van Wymelbeke V, Sulmont-Rossé C.Vieillissement, adaptation alimentaire au grand âge et petit appétit : le petit mangeur âgé. In: Hugol-Gential C, editors. Bien et Bon à Manger : Penser notre Alimentation du Quotidien à I'Institution Dijon, France: Editions Universitaires de Dijon,; 2018, p.

[68] Sulmont-Rossé C, Vandenberghe-Descamps M, Chabanet C, Van Wymelbeke $\mathrm{V}$, Feron G. Impact of culinary dependence on dietary patterns in the eldelry population. Montréal, Canada: Society for the Study of Ingestive Behavior 2017; July, 11-22.

[69] Divert C, Laghmaoui R, Crema C, Issanchou S, Van Wymelbeke V, SulmontRossé C. Improving meal context in nursing homes. Impact of four strategies on food intake and meal pleasure. Appetite 2015; 84:139-147.

[70] Sulmont-Rossé C, Gaillet M, Raclot C, Duclos M, Servelle M, Chambaron S. Impact of olfactory priming on food intake in an Alzheimer unit. Journal of Alzheimer disease 2018; 66:1497-1506.

[71] Van Wymelbeke V. Amélioration sensorielle vs amélioration environnementale sur la prise alimentaire et la satisfaction. Dijon, France: Colloque Aupalesens 2013; November 26.

[72] Service des Achats de l'Etat. Recommandation nutrition : groupe d'étude des marchés de restauration collective et nutirtion GEM-RCN, Version 2.0. 2015; https://www.economie.gouv.fr/files/directions services/daj/marches publics/oe ap/gem/nutrition/nutrition.pdf.

[73] Brownie S, Coutts R. Older Australians' perceptions and practises in relation to a healthy diet for old age: a qualitative study. Journal of Nutrition Health \& Aging 2013; 17:125-129.

[74] Rosner P, Vigier-Souvignet C. Quels atouts, obstacles et enjeux à la consommation de viande par les seniors ? 2014; http://www.interbev.fr/wpcontent/uploads/2018/07/ADIV-CIV-Rapport-Etude-SENIORS-V-finale.pdf.

[75] CLCV. Enquête sur l'alimentation des personnes âgées. 2012; http://www.clcv.org/nos-enquetes/enquete-sur-I-alimentation-des-personnesagees-02-02-2012.html

[76] Maille G.Interview de Grégoire Maille. In: Hugol-Gential C, editors. Bien et bon à manger : penser notre alimenation du quotidien à l'institution, Dijon, France: Editions Universitaires de Dijon; 2018, p.

[77] Borgstrom Bolmsjo B, Jakobsson U, Molstad S, Ostgren CJ, Midlov P. The nutritional situation in Swedish nursing homes - A longitudinal study. Arch Gerontol Geriatr 2015; 60:128-133.

[78] Maitre I, Van Wymelbeke V, Amand M, Vigneau E, Issanchou S, SulmontRosse C. Food pickiness in the elderly: Relationship with dependency and malnutrition. Disponible on-line 2014; 32:145-151. 
[79] van Nie NC, Meijers JM, Schols JM, Lohrmann C, Spreeuwenberg M, Halfens RJ. Do structural quality indicators of nutritional care influence malnutrition prevalence in Dutch, German, and Austrian nursing homes? Nutrition 2014; 30:1384-1390.

[80] Sulmont-Rossé C, Gaillet M, Raclot C, Duclos M, Servelle M, Chambaron S. Impact of olfactory priming on food intake in an Alzheimer unit. Journal of Alzheimer Disease under revision; 
Tableau 1. Distribution des participants en fonction des apports caloriques et protidiques $(\mathrm{n}=88)$.

\begin{tabular}{|c|c|c|c|c|}
\hline & \multicolumn{3}{|c|}{ Apport calorique $^{1}$} \\
\hline & & $\geq 100 \%$ & $<100$ et $\geq 66 \%$ & $<66 \%$ \\
\hline \multirow{3}{*}{$\begin{array}{l}\text { Apport } \\
\text { protidique }^{2}\end{array}$} & $\geq 100 \%$ & $13 \%$ & $3 \%$ & $0 \%$ \\
\hline & $<100$ et $\geq 66 \%$ & $16 \%$ & $22 \%$ & $8 \%$ \\
\hline & $<66 \%$ & $0 \%$ & $17 \%$ & $21 \%$ \\
\hline
\end{tabular}

${ }^{1}$ Rapport entre prise calorique observée et apport journalier conseillé (30 kcal / kg de poids corporel).

${ }^{2}$ Rapport entre prise protidique observée et apport journalier conseillé $(1,2 \mathrm{~g} / \mathrm{kg}$ de poids corporel). 
Tableau 2. Moyenne des quantités consommées par jour (en g) pour chaque catégorie d'aliment et profil de mangeur (entre parenthèses : erreur standard de la moyenne).

\begin{tabular}{|l|l|l|l|l|}
\hline & \multicolumn{1}{|c|}{$\begin{array}{c}\text { Mangeurs } \\
\text { normaux }\end{array}$} & \multicolumn{1}{|c|}{$\begin{array}{c}\text { Petits } \\
\text { mangeurs }\end{array}$} & $\begin{array}{c}\text { Tout petits } \\
\text { mangeurs }^{3}\end{array}$ & \multicolumn{1}{|c|}{$\begin{array}{c}\text { Test } \\
\text { statistique }^{4}\end{array}$} \\
\hline Produit céréalier petit-déjeuner & $99(21) a$ & $69(4) \mathrm{b}$ & $52(4) \mathrm{c}$ & $8,66^{* * *}$ \\
\hline Viande ou poisson déjeuner & $115(12) \mathrm{a}$ & $93(7) \mathrm{a}$ & $64(6) \mathrm{b}$ & $7,50^{* * *}$ \\
\hline Plat dîner & $137(23) \mathrm{a}$ & $106(10) \mathrm{a}$ & $78(9) \mathrm{b}$ & $3,59 *$ \\
\hline Fromage & $43(9)$ & $33(3)$ & $35(4)$ & 0,59 \\
\hline Produit laitier frais & $127(6)$ & $183(16)$ & $209(23)$ & 1,33 \\
\hline Soupe & $234(4)$ & $232(13)$ & $200(16)$ & 1,38 \\
\hline Fruit et légume cuits & $126(51)$ & $159(17)$ & $136(19)$ & 0,49 \\
\hline Fruit et légume crus & $175(16)$ & $109(11)$ & $98(9)$ & 2,94 \\
\hline Produit sucré aux repas & $119(30)$ & $106(12)$ & $93(9)$ & 0,60 \\
\hline Produit sucré au goûter & $23(7)$ & $37(7)$ & $37(6)$ & 0,27 \\
\hline
\end{tabular}

${ }^{1}$ Apport calorique et protidique $\geq 100 \%$.

${ }^{2}$ Apport calorique et/ou protidique $<100 \%$ mais $\geq 66 \%$.

${ }^{3}$ Apport calorique et/ou protidique $<66 \%$.

${ }^{4}$ Résultat d'une Analyse de la Variance (ANOVA) à un facteur (profil de mangeur). ${ }^{*} p<0,05 ;{ }^{* *} p<0,01$; ${ }^{* * *} p<0,001$. Pour chaque catégorie d'aliment, les moyennes associées à la même lettre ne sont pas significativement différentes au seuil de $5 \%$. 
Tableau 3. Moyenne des fréquences de consommation hebdomadaire pour chaque aliment et catégorie de dépendance des personnes âgées (entre parenthèses : erreur standard de la moyenne).

\begin{tabular}{|l|l|l|l|l|l|}
\hline & Autonome & $\begin{array}{c}\text { A domicile, } \\
\text { aide courses } \\
/ \text { repas }\end{array}$ & $\begin{array}{c}\text { A domicile, } \\
\text { portage de } \\
\text { repas }\end{array}$ & \multicolumn{1}{|c|}{ EHPAD } & \multicolumn{1}{|c|}{$\begin{array}{c}\text { Test } \\
\text { statistique }\end{array}$} \\
\hline Viande & $5,3(0,2)^{\mathrm{ab}}$ & $5,0(0,3)^{\mathrm{b}}$ & $4,8(0,3)^{\mathrm{b}}$ & $6,0(0,2)^{\mathrm{a}}$ & $3,57^{* *}$ \\
\hline Poisson & $1,7(0,1)^{\mathrm{b}}$ & $1,7(0,1)^{\mathrm{b}}$ & $2,4(0,2)^{\mathrm{a}}$ & $2,1(0,1)^{\mathrm{a}}$ & $5,85^{* * *}$ \\
\hline Jambon blanc & $1,5(0,1)^{\mathrm{b}}$ & $2,4(0,3)^{\mathrm{a}}$ & $1,7(0,2)^{\mathrm{b}}$ & $1,4(0,2)^{\mathrm{a}}$ & $4,28^{* *}$ \\
\hline CEuf & $1,7(0,1)^{\mathrm{a}}$ & $1,7(0,1)^{\mathrm{a}}$ & $1,5(0,2)^{\mathrm{ab}}$ & $1,2(0,1)^{\mathrm{b}}$ & $3,47^{*}$ \\
\hline
\end{tabular}

${ }^{4}$ Résultat d'une Analyse de la Variance (ANOVA) à trois facteurs (niveau de dépendance, sexe, âge). ${ }^{*} p<0,05 ;{ }^{* *} p<0,01 ;{ }^{* * *} p<0,001$. Pour chaque catégorie d'aliment, les moyennes associées à la même lettre ne sont pas significativement différentes au seuil de $5 \%$. 
Tableau 4. Moyenne, minimum et maximum des quantités de viande ou de poisson ingérées au déjeuner en EHPAD en situation de consommation ad libitum (entre parenthèses : erreur standard de la moyenne).

\begin{tabular}{|l|l|l|l|l|l|}
\hline \multicolumn{1}{|c|}{ Référence } & \multicolumn{1}{|c|}{ Condition } & \multicolumn{1}{c|}{ Plat protidique } & Effectif & \multicolumn{1}{c|}{$\begin{array}{c}\text { Moyenne } \\
\text { (en g) }\end{array}$} & $\begin{array}{c}\text { Min \& max } \\
\text { (en g) }\end{array}$ \\
\hline $\begin{array}{l}\text { Divert et al., } \\
{[69]^{*}}\end{array}$ & $\begin{array}{l}\text { Contrôle } \\
\text { Etude contexte }\end{array}$ & Blanquette de veau & 39 & $52(5)$ & $0-106$ \\
\hline $\begin{array}{l}\text { Divert et al., } \\
{[69]^{*}}\end{array}$ & $\begin{array}{l}\text { Contrôle } \\
\text { Etude condiment }\end{array}$ & Filet de merlu & 37 & $65(6)$ & $0-110$ \\
\hline $\begin{array}{l}\text { Divert et al., } \\
{[69]^{*}}\end{array}$ & $\begin{array}{l}\text { Contrôle } \\
\text { Etude intitulé des } \\
\text { plats }\end{array}$ & Sauté de porc & 39 & $51(6)$ & $0-105$ \\
\hline $\begin{array}{l}\text { Divert et al., } \\
\text { [69] }\end{array}$ & $\begin{array}{l}\text { Contrôle } \\
\text { Etude taille de } \\
\text { portion }\end{array}$ & Pintade en sauce & 37 & $50(5)$ & $0-103$ \\
\hline $\begin{array}{l}\text { Sulmont et al., } \\
{[80]}\end{array}$ & Contrôle I & Rôti de porc & 34 & $65(8)$ & $0-156$ \\
\hline $\begin{array}{l}\text { Sulmont et al., } \\
{[80]}\end{array}$ & Contrôle II & Rôti de porc & 34 & $73(8)$ & $0-203$ \\
\hline $\begin{array}{l}\text { Van } \\
\text { Wymelbeke et } \\
\text { al., [71] }\end{array}$ & Contrôle I & Blanquette de veau & 75 & $69(4)$ & $0-163$ \\
\hline $\begin{array}{l}\text { Van } \\
\text { Wymelbeke et } \\
\text { al., [71] }\end{array}$ & Contrôle II & Blanquette de veau & 78 & $65(4)$ & $0-181$ \\
\hline
\end{tabular}

* Ce article présente 4 études différentes (impact du contexte, impact de condiments, impact de l'intitulé des plats et étude de la taille des portions) sur la prise alimentaire en EHPAD. Chaque étude comportait une condition contrôle (présentée ici) et deux conditions expérimentales (non présentées ici). 
Tableau 5. Analyse des motifs de changement dans les consommations avec l'âge. Dès lors qu'une personne reportait un changement de consommation par rapport à l'âge de 30-40 ans, elle devait indiquer la raison de ce changement (plusieurs réponses possibles). Le tableau présente le pourcentage de citations associés à chaque motif de changement pour une diminution de consommation de viande rouge ou de viande blanche ainsi que pour une augmentation de consommation de volaille ou de poisson.

\begin{tabular}{|c|c|c|c|c|c|c|c|c|}
\hline \multirow[b]{2}{*}{ Aliment } & \multicolumn{4}{|c|}{ Personnes âgées autonomes ( $n=463)$} & \multicolumn{4}{|c|}{$\begin{array}{l}\text { Personnes âgées dépendantes } \\
\text { pour leur alimentation }(n=196)\end{array}$} \\
\hline & $\begin{array}{l}\text { Viande } \\
\text { rouge* }\end{array}$ & $\begin{array}{c}\text { Viande } \\
\text { blanche* }\end{array}$ & Volaille & Poisson & $\begin{array}{l}\text { Viande } \\
\text { rouge* }\end{array}$ & $\begin{array}{c}\text { Viande } \\
\text { blanche* }\end{array}$ & Volaille & Poisson \\
\hline $\begin{array}{l}\text { Evolution de la } \\
\text { consommation }\end{array}$ & Moins & Moins & Plus & Plus & Moins & Moins & Plus & Plus \\
\hline Nombre de réponses & 281 & 156 & 137 & 210 & 126 & 73 & 49 & 69 \\
\hline \begin{tabular}{|l|}
$\begin{array}{l}\text { Evolution des } \\
\text { préférences }\end{array}$ \\
\end{tabular} & 42 & 35 & 31 & 50 & 18 & 16 & 6 & 32 \\
\hline Raison de santé & 26 & 24 & 28 & 40 & 7 & 8 & 6 & 3 \\
\hline Menu imposé & 1 & 1 & 2 & 1 & 63 & 59 & 86 & 70 \\
\hline Baisse d'appétit & 17 & 21 & 0 & 0 & 18 & 22 & 0 & 0 \\
\hline Surveillance du poids & 3 & 3 & 7 & 7 & 1 & 3 & 0 & 1 \\
\hline \begin{tabular}{|l|} 
Raison liée à des \\
problèmes de dentition
\end{tabular} & 7 & 6 & 0 & 2 & 8 & 5 & 0 & 1 \\
\hline $\begin{array}{l}\text { Raison liée à la } \\
\text { praticité des aliments }\end{array}$ & 3 & 6 & 33 & 27 & 2 & 3 & 6 & 13 \\
\hline \begin{tabular}{|l|} 
Raison liée à la \\
qualité des aliments
\end{tabular} & 7 & 10 & 12 & 8 & 6 & 11 & 2 & 1 \\
\hline Préférence du conjoint & 4 & 1 & 4 & 4 & 0 & 0 & 0 & 0 \\
\hline Raison financière & 7 & 8 & 10 & 2 & 1 & 0 & 4 & 1 \\
\hline $\begin{array}{l}\text { Changement dans la } \\
\text { composition du foyer }\end{array}$ & 10 & 12 & 3 & 4 & 2 & 4 & 2 & 1 \\
\hline \begin{tabular}{|l} 
Raison liée à \\
l'environnement
\end{tabular} & 1 & 1 & 0 & 0 & 0 & 0 & 0 & 0 \\
\hline Autre & 7 & 7 & 7 & 10 & 5 & 7 & 0 & 0 \\
\hline
\end{tabular}

*Viande rouge : bœuf, mouton, cheval ; Viande blanche : veau, porc. 
Figure 1. Distribution des quantités consommées de blanquette de veau au déjeuner dans l'étude de Van Wymelbeke et al. [71].

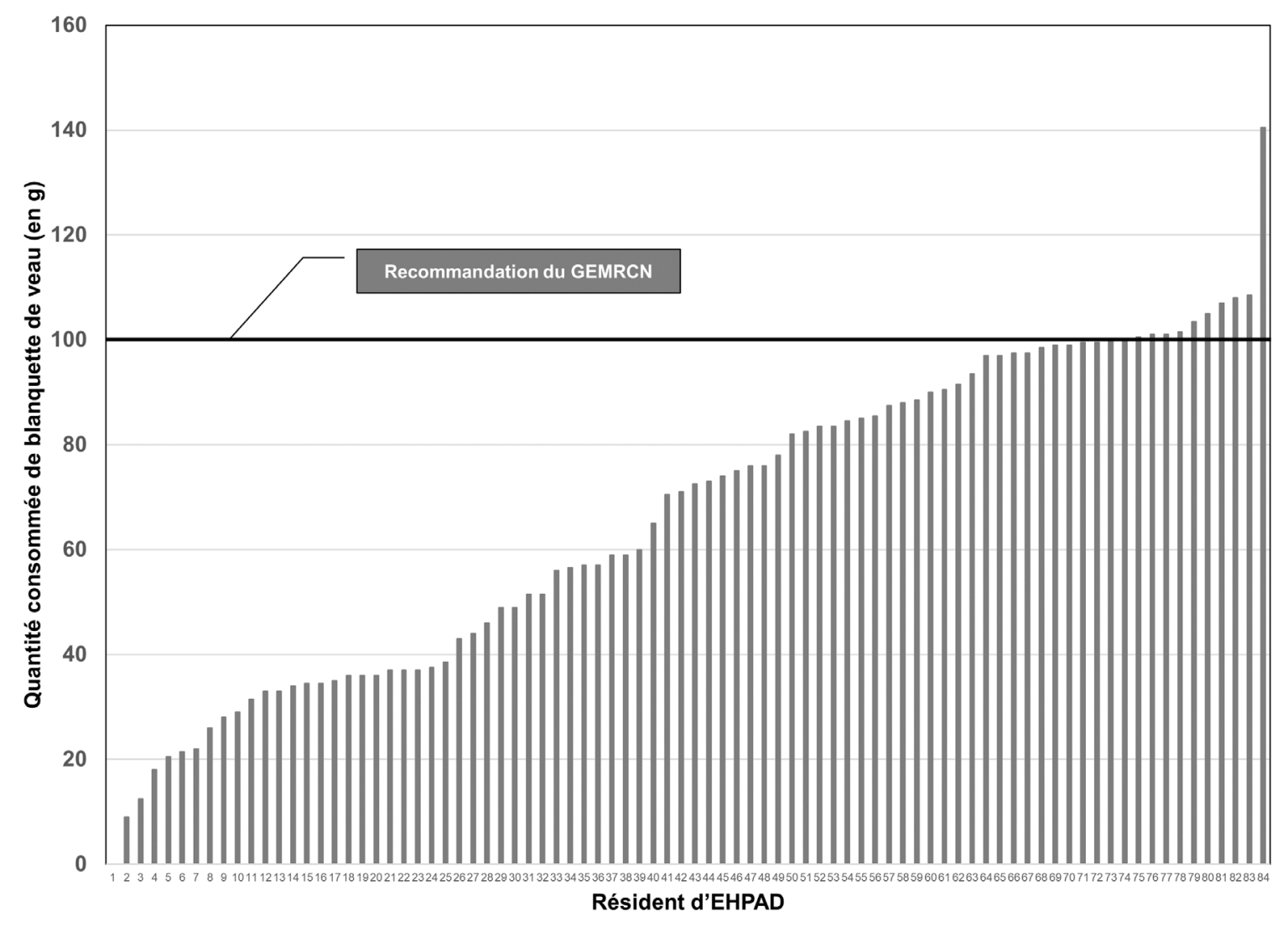


Figure 2. Evolution des consommations avec l'âge pour les personnes âgées autonomes pour leur alimentation $(n=463$, Figure A) et pour les personnes âgées dépendantes pour leur alimentation ( $n=196$, Figure B). Pour chaque catégorie d'aliments, les participants ont répondu à la question « Diriez-vous que maintenant vous mangez moins, autant ou plus de cet aliment que lorsque vous aviez 30-40 ans?». La figure présente le pourcentage de personnes ayant respectivement répondu "moins", "autant" ou "plus" pour chaque catégorie d'aliment.

\section{A. Personnes âgées autonomes}

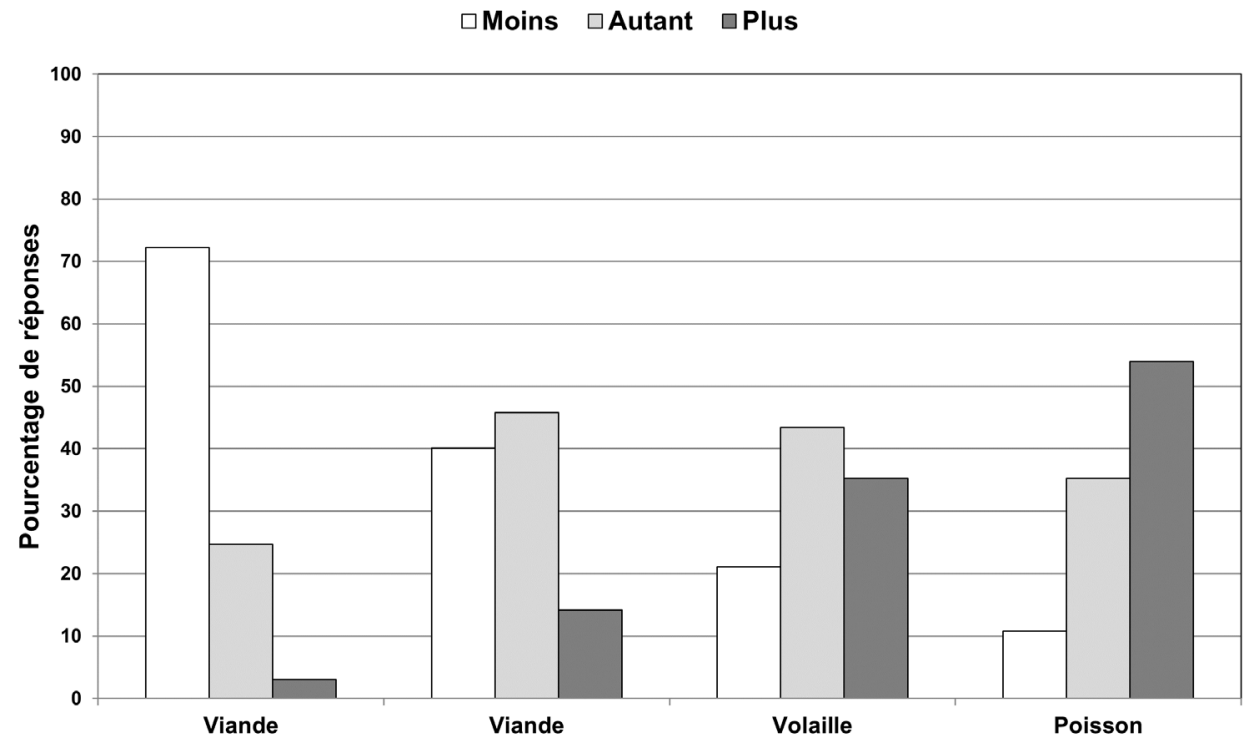

B. Personnes âgées dépendantes pour leur alimentation

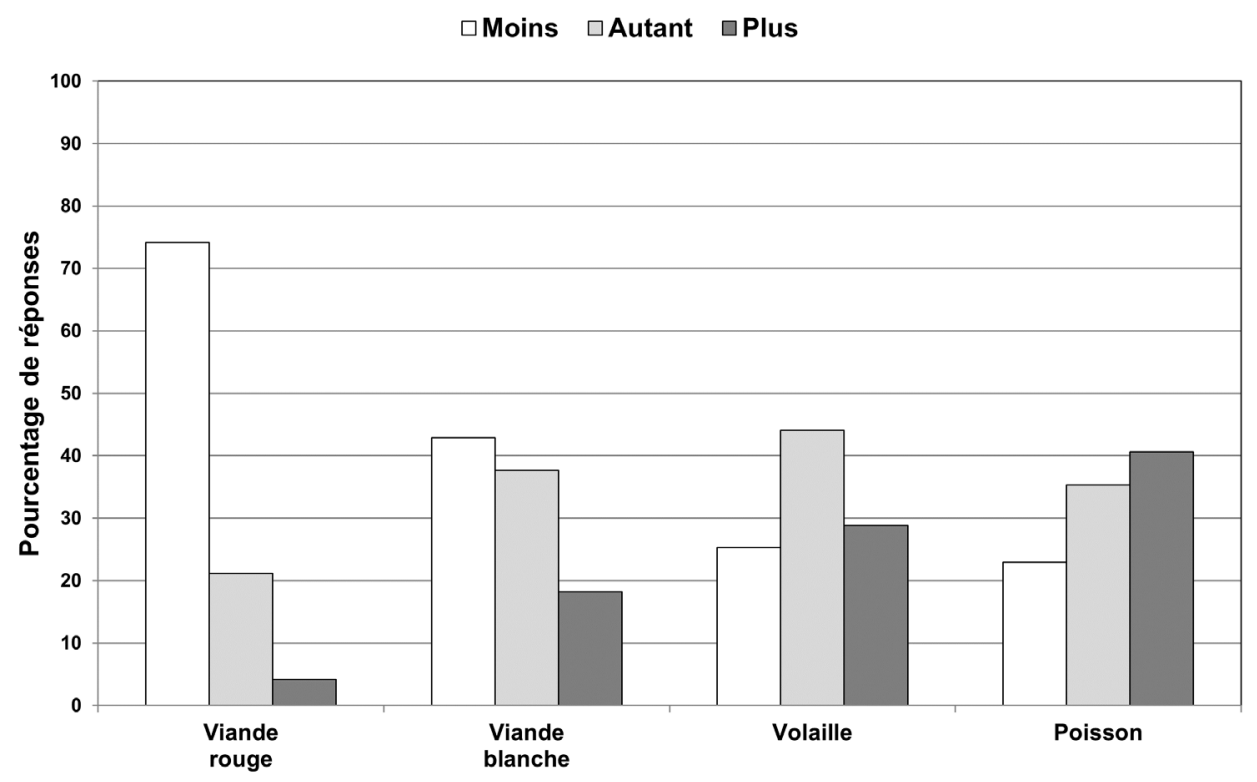

